\title{
ON PATH FOLLOWING CONTROL OF NONHOLONOMIC MOBILE MANIPULATORS
}

\author{
ALICJA MAZUR, DAWID SZAKIEL \\ Institute of Computer Engineering, Control and Robotics \\ Wrocław University of Technology, ul. Janiszewskiego 11/17, 50-372 Wrocław, Poland \\ e-mail: alicja.mazur@pwr.wroc.pl
}

\begin{abstract}
This paper describes the problem of designing control laws for path following robots, including two types of nonholonomic mobile manipulators. Due to a cascade structure of the motion equation, a backstepping procedure is used to achieve motion along a desired path. The control algorithm consists of two simultaneously working controllers: the kinematic controller, solving motion constraints, and the dynamic controller, preserving an appropriate coordination between both subsystems of a mobile manipulator, i.e. the mobile platform and the manipulating arm. A description of the nonholonomic subsystem relative to the desired path using the Frenet parametrization is the basis for formulating the path following problem and designing a kinematic control algorithm. In turn, the dynamic control algorithm is a modification of a passivity-based controller. Theoretical deliberations are illustrated with simulations.
\end{abstract}

Keywords: mobile manipulator, nonholonomic constraints, path following, Frenet parametrization.

\section{Introduction}

In the paper, new control algorithms for a special class of robotic objects, namely, nonholonomic mobile manipulators, are presented. A mobile manipulator is defined as a robotic system composed of a mobile platform and a manipulator mounted on the platform equipped with nondeformable wheels. Such a combined system is able to perform manipulation tasks in a much larger workspace than a fixed-base manipulator. The downside of such design is more complex modeling and control.

First, the dynamics of the combined system are much more complicated because they include dynamic interactions between the mobile platform and the manipulator. This can be observed, e.g., when the movement of the manipulator implies the movement of the platform, even if platform actuators do not work. Second, the existence of nonholonomic constraints implies a nontrivial form of the kinematics of the mobile manipulator. Third, a particular point in the workspace can be reached either by moving the manipulator or the mobile platform, or by coordinated motion of both. This means that the solution to the tracking problem is not unique.

The problem of designing a control law for mobile manipulators has recently received much attention. There have appeared many works presenting different methods of solving this control problem for mobile manipulators. In the literature, two general groups of mobile manipulator control strategies can be found. Within the first group, decentralized control strategies have been developed. All subsystem controllers were designed separately and, subsequently, the interactions between the platform and the manipulator needed to be considered and compensated (Chung et al., 1998; Huang et al., 1998). Within the second group, a unified control for both parts constituting a mobile manipulator is developed.

The problem with the definition of the path for a complete mobile manipulator, without decomposition, is that the behavior of subsystems is unpredictable, because the same path defined in global coordinates can be executed by separate subsystems or by both of them. Sometimes, it is important to move the platform and simultaneously unload a payload-such a task is defined relative to the base of the manipulator mounted on the platform (a definition relative to the end-effector is ill conditioned). In such a situation the decomposition of the task into tasks defined separately for both subsystems is more natural and convenient (Mazur, 2004).

The most popular approach to the control of mobile manipulators explores the idea of input-output decoupling and linearization. In (Yamamoto and Yun, 1994; 1996), the authors used the concept of the manipulability ellip- 
soid for a two link planar mobile manipulator. This criterion (kinematic or dynamic) can select motion directions which are most promising. These directions have more maneuver possibilities to execute the desired task. The authors applied input-output decoupling and linearization to a two-link planar mobile manipulator subject to nonholonomic constraints in the platform and developed a coordination algorithm based on the concept of a preferred operating region. The control task was to move the object using only the mobile platform and the robotic arm had to achieve a constant configuration with maximal manipulability. In (Tan et al., 2003; Khatib, 1999), inputoutput decoupling was the point of departure to design a hybrid strategy preserving motion/force control but only for mobile manipulators with holonomic platforms. For the case when some uncertainties in the dynamics of nonholonomic mobile manipulator appear, in (Dong, 2002) the author discussed a trajectory and force tracking control problem subject to unknown inertia parameters and proposed adaptive controllers based on a suitable reduced dynamic model. In turn, in (Li et al., 2007), another adaptive strategy for parameter uncertainty was presented.

A different approach to centralized control can be found in (Tchoń et al., 2004; Tchoń and Jakubiak, 2004), where an endogenous configuration space approach was presented. In these works, kinematics were treated as a map from a Hilbert space $H_{\infty}$ of controls and manipulator joint positions into a task-space of the mobile manipulator. Such an approach makes it possible to move a mobile manipulator from a start to an end point, but without specifying desired configurations of both subsystems. This means that the posture of the platform (forward or backward motion) is out of control. The limitation of this method is that it generates control only for a point stabilization task in fixed time.

In real applications, it is necessary to take into consideration the dynamics of the object. The crucial point is the evaluation of stability, e.g., using the ZMP (zero moment point) method. ZMP is a special point where resultant moments of gravity, the inertial forces of the mobile manipulator and the external forces are zero. In (Hatano and Obara, 2003), the authors studied the stability of transitional states and formulated criteria for stable motion. This method can be used to prove the stability of the mobile manipulator's motion.

In the present paper, we define a path following problem for the mobile manipulator in a different way, see (Mazur, 2004). The desired task is decomposed into two separate subtasks defined for each subsystem independently: the end-effector has to follow a desired geometric path described relative to the base of the robotic arm (i.e., relative to the platform), and the task of the platform is to follow a desired curve lying on a plane. Such a formulation of the task makes it possible to successively unload the payload transported by the mobile manipulator during the control process.

Taking into account the type of mobility of components, there are four possible configurations: type $(h, h)$ - both the platform and the manipulator are holonomic, type $(h, n h)$ - a holonomic platform with a nonholonomic manipulator, type $(n h, h)$-a nonholonomic platform with a holonomic manipulator, and type $(n h, n h)$ - both the platform and the manipulator are nonholonomic. The notion doubly nonholonomic manipulator was introduced in (Tchon et al., 2004) for the type $(n h, n h)$. The path following problem formulated for a doubly nonholonomic mobile manipulator has not been considered in the literature so far.

\section{General model of the nonholonomic mobile manipulator}

2.1. Nonholonomic constraints. A hypothetical mobile manipulator is presented in Fig. 1.

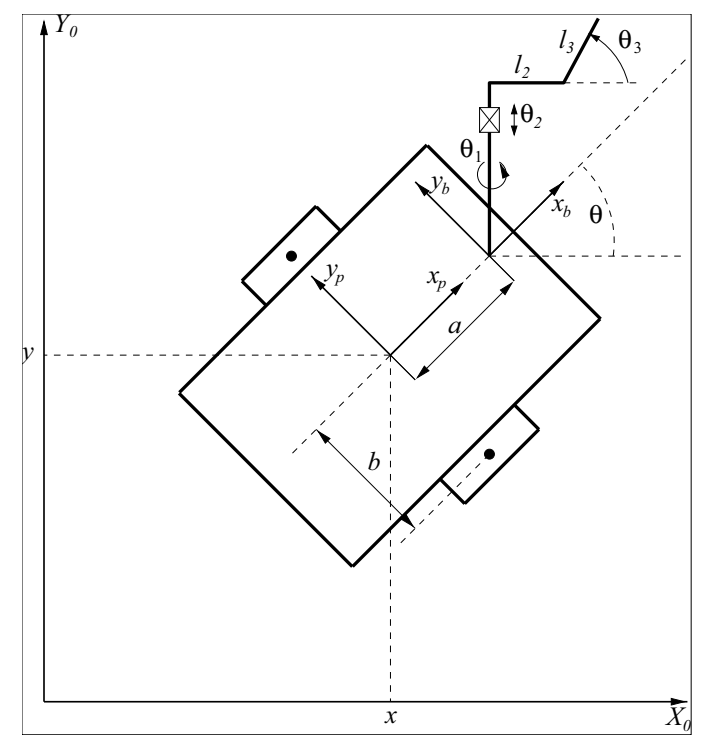

Fig. 1. RTR manipulator on a mobile platform of the $(2,0)$ class.

2.1.1. Nonholonomic constraints for a wheeled mobile platform. The motion of the mobile platform can be described by generalized coordinates $q_{m} \in \mathbb{R}^{n}$ and generalized velocities $\dot{q}_{m} \in \mathbb{R}^{n}$. The wheeled mobile platform should move without the slippage of its wheels. This is equivalent to the assumption that momentary velocity at the contact point between each wheel and the motion plane is equal to zero. This assumption implies the existence of $l(l<n)$ independent nonholonomic constraints expressed in the Pfaffian form,

$$
A\left(q_{m}\right) \dot{q}_{m}=0,
$$

where $A\left(q_{m}\right)$ is a full rank $l \times n$ matrix. 
Since, due to (1), the platform velocity is in the null space of $A\left(q_{m}\right)$, it is always possible to find a vector of special auxiliary velocities $\eta \in \mathbb{R}^{m}, m=n-l$, such that

$$
\dot{q}_{m}=G\left(q_{m}\right) \eta,
$$

where $G$ is a full rank $n \times m$ matrix satisfying the relationship $A G=0$. In (Canudas de Wit et al., 1996), it was shown that all wheeled mobile platforms can be divided, according to the motion possibilities given by (2), into special classes, defined by the following notions:

- degree of mobility $\sigma_{m}$ - the dimension of the available space in the contact point between wheels and a surface,

$$
1 \leq \sigma_{m} \leq 3
$$

- degree of steerability $\sigma_{s}$ - the number of independently driven steering wheels of the platform,

$$
0 \leq \sigma_{s} \leq 2
$$

An additional condition, which the parameters $\sigma_{m}$ and $\sigma_{s}$ have to fulfill, is

$$
2 \leq \sigma_{m}+\sigma_{s} \leq 3
$$

From the above inequalities it results that there exist only five classes of wheeled mobile platforms, presented in Table 1 Defining a class for the platform, the parameters $\left(\sigma_{m}, \sigma_{s}\right)$ have to be pointed out.

Table 1. Possible classes of wheeled mobile platforms.

\begin{tabular}{|c|c|c|c|c|c|}
\hline$\sigma_{m}$ & 3 & 2 & 2 & 1 & 1 \\
\hline$\sigma_{s}$ & 0 & 0 & 1 & 2 & 1 \\
\hline
\end{tabular}

Apart from the $(3,0)$ class, which is holonomic, the remaining classes are nonholonomic, i.e., they realize motion with restricted mobility (D'Andréa-Novel et al., 1991).

2.1.2. Nonholonomic constraints for a manipulator. A rigid manipulator can be a holonomic or a nonholonomic system-it depends on construction of its drives. In (Nakamura et al., 2001), the authors presented a new nonholonomic mechanical gear, which was able to transmit velocities from the inputs to many passive joints, see Figs. 2 and 3 The nonholonomic constraints in the gear appear by assumption on rolling contact without slippage between balls of gear and special supporting wheels in the robot joints.

The basic components of the gear presented in Fig. 2 are a ball and three wheels-an input wheel IW and two output wheels $\mathrm{OW}_{1}$ and $\mathrm{OW}_{2}$. The velocity constraints of the ball are only due to point contact with the wheels. The

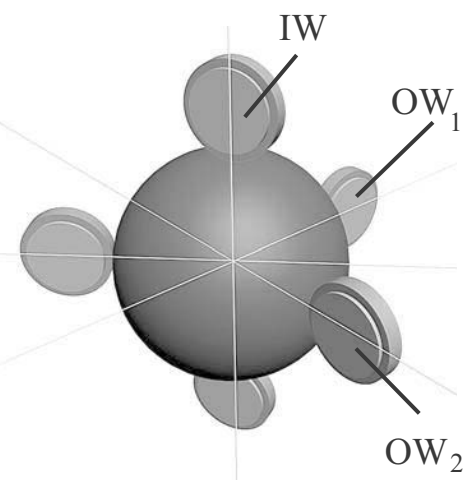

Fig. 2. Schematic of the nonholonomic gear.

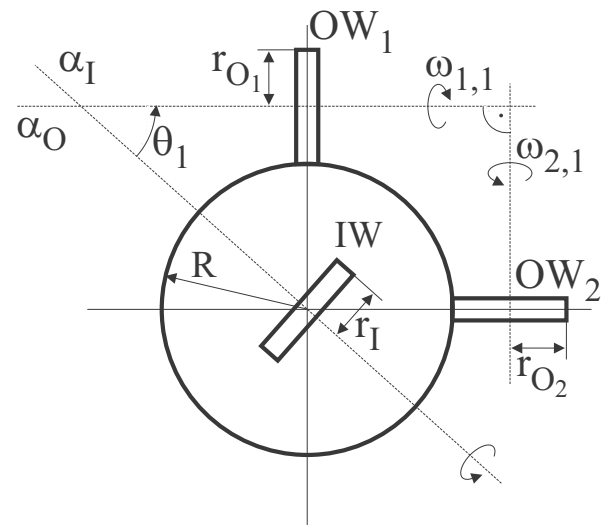

Fig. 3. Nonholonomic gear seen from above.

input wheel IW with the radius $r_{I}$ is located on a pole of the ball (with the radius $R$ ). The input wheel is mounted in the first joint, and the output wheels are mounted in the next joint. The wheel IW rotates around the fixed axis $\alpha_{I}$ with an angular velocity $u_{2}$, which plays the role of a control input. The rotating input wheel makes the ball rotate. The next elements of the gear are two output wheels with the radii $r_{O_{1}}$ and $r_{O_{2}}$, located in the ball's equator. The wheel $\mathrm{OW}_{1}$ rotates around an axis $\alpha_{O}$, which, together with the axis of the input wheel, forms a joint angle $\theta_{1}$. The angular velocity $\dot{\theta}_{1}=u_{1}$ is the second control input for the manipulator with nonholonomic gears. The rotation axis of the wheel $\mathrm{OW}_{2}$ is perpendicular to the axis $\alpha_{O}$.

In (Nakamura et al., 2001), the following kinematic equations for a nonholonomic $n$-pendulum can be found:

$$
\begin{aligned}
& \dot{\theta}_{1}=u_{1}, \\
& \dot{\theta}_{i}=a_{i} \sin \theta_{i-1} \prod_{j=1}^{i-2} \cos \theta_{j} u_{2}, \quad i \in\{2, \ldots, n\},
\end{aligned}
$$

with positive coefficients $a_{i}$, depending on gear ratios.

It can be noticed that with only two control inputs $u_{1}$ and $u_{2}$ it is possible to control many joints of the non- 
holonomic manipulator equipped with gears designed by Nakamura et al. (2001).

2.2. Dynamic equations of a nonholonomic mobile manipulator. The mobile manipulator is a system which consists of a wheeled mobile platform and a rigid onboard manipulator. In this paper, only two types of mobile manipulators are considered. In the $(n h, h)$ type, the mobile platform is nonholonomic and the manipulating arm is an ordinary rigid manipulator. In the $(n h, n h)$ type, the wheeled platform and the manipulator are nonholonomic subsystems. The nonholonomic constraints are based on the no-slippage assumption in the gear or in the contact point between each wheel and a surface. To obtain a mathematical description of a nonholonomic control system, the d'Alembert principle must be used. We consider the dynamics for each type of mobile manipulator separately, because they are expressed in different variables.

2.2.1. Dynamic equations of the mobile manipulator of the $(\boldsymbol{n} \boldsymbol{h}, \boldsymbol{h})$ type. Let the vector of generalized coordinates of the mobile manipulator be denoted by $q=$ $\left(q_{m}^{T}, q_{r}^{T}\right)^{T}$, where $q_{m}$ defines a vector of mobile platform coordinates and $q_{r} \in \mathbb{R}^{p}$ denotes a vector of joint coordinates of the rigid manipulator with $p$ degrees of freedom,

$$
q_{r}=\left(\theta_{1}, \theta_{2}, \ldots, \theta_{p}\right)^{T} .
$$

Because of the nonholonomy of constraints, for obtaining the dynamic model of the mobile manipulator, the d'Alembert principle has to be used,

$$
Q(q) \ddot{q}+C(q, \dot{q}) \dot{q}+D(q)=A_{1}\left(q_{m}\right) \lambda+B \tau,
$$

where $Q(q)$ is the inertia matrix of the mobile manipulator, $C(q, \dot{q})$ is the matrix of Coriolis and centrifugal forces, $D(q)$ is the vector of gravitational terms, $A_{1}$ is the matrix of nonholonomic constraints, $\lambda \in \mathbb{R}^{l}$ is the vector of Lagrange multipliers, $B$ is the input matrix, $\tau=\left(\tau_{m}^{T}, \tau_{r}^{T}\right)$ is the vector of controls. The above model of dynamics can be expressed in a block matrix form as

$$
\begin{gathered}
{\left[\begin{array}{cc}
Q_{11} & Q_{12} \\
Q_{21} & Q_{22}
\end{array}\right]\left(\begin{array}{c}
\ddot{q}_{m} \\
\ddot{q}_{r}
\end{array}\right)+\left[\begin{array}{cc}
C_{11} & C_{12} \\
C_{21} & C_{22}
\end{array}\right]\left(\begin{array}{c}
\dot{q}_{m} \\
\dot{q}_{r}
\end{array}\right)+\left(\begin{array}{c}
0 \\
D_{2}
\end{array}\right)} \\
=\left[\begin{array}{c}
A^{T} \\
0
\end{array}\right] \lambda+\left[\begin{array}{cc}
B_{1} & 0 \\
0 & I
\end{array}\right]\left(\begin{array}{c}
\tau_{m} \\
\tau_{r}
\end{array}\right),
\end{gathered}
$$

where $A$ is the Pfaffian matrix given by (1), $D_{2}$ is a vector of gravity for the robotic arm, $B_{1}$ defines coordinates of the mobile platform directly controllable by the actuators, $\tau_{m}$ is a vector of input forces or torques applied to the mobile platform, and $\tau_{r}$ is a vector of input forces for the rigid onboard manipulator. Equation (6) will be called the dynamics of the mobile manipulator expressed in generalized coordinates.
Now we want to express the model of dynamics using the auxiliary velocities (2) for the mobile platform. We compute

$$
\ddot{q}_{m}=G\left(q_{m}\right) \dot{\eta}+\dot{G}\left(q_{m}\right) \eta
$$

and eliminate the vector of Lagrange multipliers (using the condition $G^{T} A^{T}=0$ ) by premultiplication of the mobile platform equations by the matrix $G^{T}$. After substituting for $\dot{q}_{m}$ and $\ddot{q}_{m}$, we get

$$
Q^{*}\left(\begin{array}{c}
\dot{\eta} \\
\ddot{q}_{r}
\end{array}\right)+C^{*}\left(\begin{array}{c}
\eta \\
\dot{q}_{r}
\end{array}\right)+D^{*}=B^{*} \tau,
$$

where

$$
\begin{aligned}
Q^{*} & =\left[\begin{array}{cc}
G^{T} Q_{11} G & G^{T} Q_{12} \\
Q_{21} G & Q_{22}
\end{array}\right], \\
C^{*} & =\left[\begin{array}{cc}
G^{T}\left(C_{11} G+Q_{11} \dot{G}\right) & G^{T} C_{12} \\
Q_{21} \dot{G}+C_{21} G & C_{22}
\end{array}\right], \\
D^{*} & =\left(\begin{array}{c}
0 \\
D_{2}
\end{array}\right), \\
B^{*} & =\left[\begin{array}{cc}
G^{T} B_{1} & 0 \\
0 & I
\end{array}\right] .
\end{aligned}
$$

The above model of mobile manipulator dynamics will be the point of departure for designing a dynamic control algorithm. It should be noted that the complete system consists of the dynamic model (7) and the purely kinematic relationship (2).

\subsubsection{Dynamic equations of a mobile manipulator of} the $(\boldsymbol{n h}, \boldsymbol{n h})$ type. In the case of a mobile manipulator of the $(n h, n h)$ type, both subsystems are nonholonomic. Therefore, the dynamics expressed in generalized coordinates due to the d'Alembert principle have the form

$$
Q(q) \ddot{q}+C(q, \dot{q}) \dot{q}+D(q)=A_{1} \lambda_{1}+A_{2} \lambda_{2}+B \tau,
$$

where $A_{1}$ is the matrix of nonholonomic constraints for the wheeled mobile platform, $\lambda_{1}$ is the vector of Lagrange multipliers for the platform, $A_{2}$ is the matrix of nonholonomic constraints for the manipulator, $\lambda_{2}$ is the vector of Lagrange multipliers for the manipulator, $B$ is the input matrix, $\tau=\left(\tau_{m}^{T}, \tau_{r}^{T}\right)$ is the vector of controls. The matrices $A_{1}, A_{2}$ and $B$ are defined as follows:

$$
\begin{gathered}
A_{1}=\left(\begin{array}{c}
A_{11}^{T}\left(q_{m}\right) \\
0
\end{array}\right), \quad A_{2}=\left(\begin{array}{c}
0 \\
A_{22}^{T}\left(q_{r}\right)
\end{array}\right), \\
B=\left[\begin{array}{cc}
B_{11} & 0 \\
0 & B_{22}
\end{array}\right],
\end{gathered}
$$

where $A_{11}\left(q_{m}\right)$ is the Pfaffian matrix for the mobile platform and $A_{2}\left(q_{r}\right)$ is the Pfaffian matrix for the nonholonomic manipulator. The submatrices $B_{11}$ and $B_{22}$ describe which coordinates of the platform and the manipulator are directly driven by actuators. 
For the $(n h, n h)$ mobile manipulator, the nonholonomic constraints hold for each subsystem. These constraints can be expressed as driftless control systems and described as a single kinematic equation,

$$
\dot{q}=\left(\begin{array}{c}
\dot{q}_{m} \\
\dot{q}_{r}
\end{array}\right)=\left[\begin{array}{cc}
G_{1} & 0 \\
0 & G_{2}
\end{array}\right]\left(\begin{array}{l}
\eta \\
u
\end{array}\right)=G \zeta,
$$

where $\zeta$ is a vector of auxiliary velocities for both subsystems. Substituting the expression (9) into the dynamics (8), we obtain

$$
Q^{*} \dot{\zeta}+C^{*} \zeta+D^{*}=B^{*} \tau
$$

with elements defined in the following way:

$$
\begin{aligned}
Q^{*} & =G^{T} Q G, \\
C^{*} & =G^{T}(Q \dot{G}+C G), \\
D^{*} & =G^{T} D, \\
B^{*} & =G^{T} B .
\end{aligned}
$$

Equation (10) describes the dynamics of the doubly nonholonomic mobile manipulator expressed in auxiliary velocities.

It is worth mentioning that a mobile manipulator with a wheeled platform has a special property, which does not hold for its subsystems (Dulęba, 2000).

Property 1. For a mobile manipulator with a wheeled nonholonomic mobile platform, a skew-symmetry between the inertia matrix $Q^{*}$ and the matrix of Coriolis and the centrifugal forces $C^{*}$ does not hold anymore. To regain the skew-symmetry, a special nontrivial correction matrix $C_{K}$ must be added,

$$
\frac{\mathrm{d}}{\mathrm{d} t} Q^{*}=\left(C^{*}+C_{K}\right)+\left(C^{*}+C_{K}\right)^{T} .
$$

Any matrix which fulfills the relation 111 can play the role of a correction matrix. The following expression describing the form of a $C_{K}$ matrix:

$$
C_{K}=C_{K}^{T}=\frac{1}{2}\left\{Q^{*}-C^{*}-\left(C^{*}\right)^{T}\right\}
$$

should be calculated before starting the regulation process. Arguments of the correction matrix are the generalized coordinates and velocities of a mobile manipulator, which can be measured in every moment and used during the control process.

\section{Control problem statement}

Our goal is to find a control law guaranteeing path following for nonholonomic mobile manipulators. As mentioned in Section 1, the desired task for the mobile manipulator can be decomposed into two independent parts: the endeffector of the robotic arm has to follow a geometric path

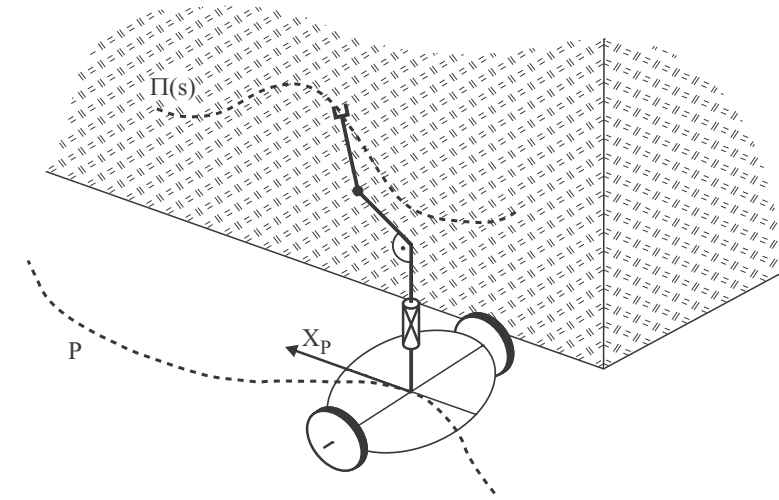

Fig. 4. Desired path for a mobile manipulator: $P(s)$-desired path for the platform, $\Pi(s)$-desired path for the manipulator.

$\Pi(s)$ described relative to its base (i.e., relative to the platform), and the task of the platform is to follow a desired curve $P(s)$ lying on the plane, see Fig. 4.

The problem of path following is defined in different manner for both types of nonholonomic mobile manipulators considered:

- $(n h, h)$ mobile manipulator-because sizes of workspaces for each subsystem are considerably different, the manipulator's end-effector should follow the geometric path described in Cartesian coordinates, and it should stop at the end of the desired path $\Pi(s)$. In turn, the wheeled nonholonomic platform should move continuously along the desired curve.

- $(\boldsymbol{n h}, \boldsymbol{n h})$ mobile manipulator-each subsystem is nonholonomic, therefore the manipulator's endeffector should follow the geometric path described in Cartesian coordinates without stopping at the end of the desired path and the wheeled mobile platform should move continuously along desired path $P(s)$.

A goal of this section will be to address the following control problem for mobile manipulators:

Design a control law $\tau$ and a path parametrization $s=s(t)$ (only for the holonomic robotic arm) such that a mobile manipulator with fully known dynamics follows the desired paths defined separately for each subsystem, and tracking errors converge asymptotically to zero.

Additionally, we assume that the desired paths are smooth curves which have two smooth and bounded time derivatives.

Note that a complete model of the nonholonomic system has the structure of two cascaded equations: kinematics (nonholonomic constraints) and dynamics. For this reason, the backstepping-like procedure for the designing of control law should be used (Krstić et al., 1995): 
- Kinematic controller $\eta_{r}(t)$ or $\zeta_{r}(t)$ : it represents an embedded control input, which ensures the realizability of path following for nonholonomic constraints. The kinematic controller can be treated as a solution to the kinematics (2) or (9), if the dynamics were not present. Such a controller generates a 'velocity profile', which can be executed in practice. The convergence of the kinematic control algorithm must be proven.

- Dynamic controller $\tau$ : as a consequence of the cascaded structure of the model, the system's auxiliary velocities $\eta$ or $\zeta$ cannot be commanded directly, as assumed in the design of kinematic control, and instead they must be realized as the output of the dynamics (7) or (10) driven by $\tau$. The dynamic input $\tau$ intends to regulate the real velocities $\eta$ or $\zeta$ toward the reference control $\eta_{r}$ or $\zeta_{r}$ and, therefore, it attempts to provide control input necessary to achieve the desired task.

Since there exists a difference between the real velocities $\eta$ or $\zeta$ of the nonholonomic mobile manipulator and the reference control $\eta_{r}$ or $\zeta_{r}$, it is necessary to take into account the influence of the errors $e_{\eta}=\eta-\eta_{r}$ or $e_{\zeta}=\zeta-\zeta_{r}$ on the behavior of the full mathematical model describing the nonholonomic mobile manipulator.

\section{Path following problem for subsystems of a mobile manipulator}

4.1. Path following problem for a nonholonomic subsystem. In this section, equations describing the motion of a nonholonomic system in the task-space relative to a given curve, which the system should follow, are derived. First, we discuss the path following problem for nonholonomic mobile platforms and give a detailed solution for a mobile platform of the $(2,0)$ class (unicycle) due to the Samson control algorithm. Next, we adapt the Frenet parametrization for the nonholonomic manipulator with gears designed by Nakamura, et al. (2001). We use such parametrization to obtain a kinematic control algorithm for a nonholonomic manipulation arm.

4.1.1. Path following problem for a nonholonomic wheeled mobile platform. The mobile platform and the path to be followed are presented in Fig. 5 The path $P$ is characterized by a curvature $c(s)$, which is an inversion of the radius of the circle tangent to the path at a point characterized by the parameter $s$. Consider a moving point $\mathrm{M}$ and the associated Frenet frame defined on the curve $P$ by the normal and tangent unit vectors $x_{n}$ and $\mathrm{d} r / \mathrm{d} s$. The point $\mathrm{M}$ is the mass center of a mobile platform and $\mathrm{M}^{\prime}$ is the orthogonal projection of the point $\mathrm{M}$ on the path $P$.

The point M' exists and is uniquely defined if the following conditions are satisfied (Fradkov et al., 1999):

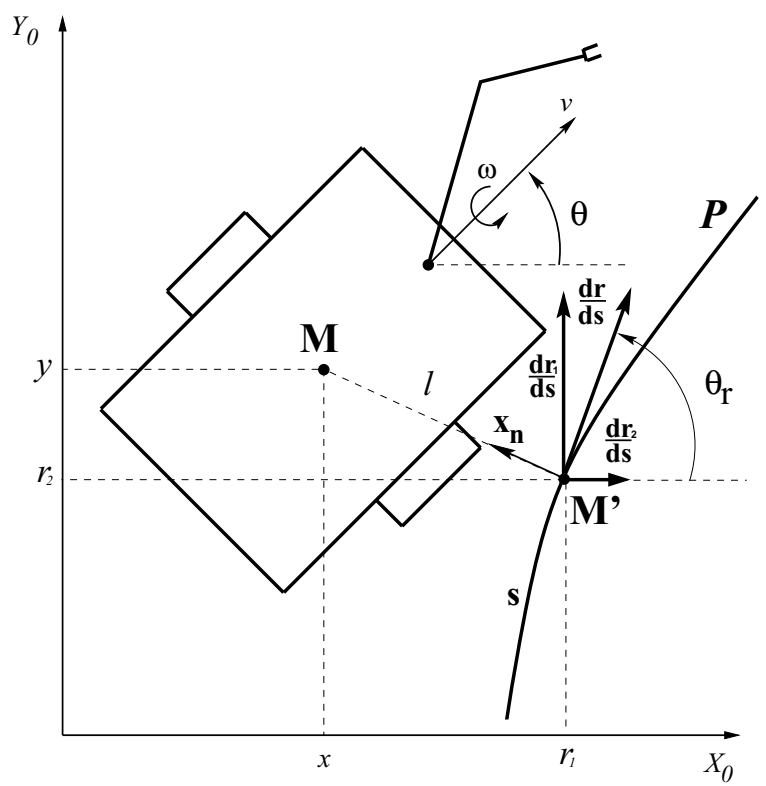

Fig. 5. Illustration of the path following problem for a nonholonomic platform.

- The radius of any circle tangenting $P$ at two or more points whose interior does not contain any point of the curve is lowerbounded by some positive real number denoted as $r_{\min }$.

This assumption implies in particular that the curvature $c(s)$ does not exceed $1 / r_{\min }$.

- Under this assumption, if the distance between the path $P$ and the point $\mathrm{M}$ is smaller than $r_{\min }$, there is a unique point on $P$ denoted by $\mathrm{M}^{\prime}$.

The coordinates of the point $\mathrm{M}$ relative to the Frenet frame are $(0, l, 0)$ and those relative to the basic frame $X_{0} Y_{0} Z_{0}$ are equal to $(x, y, 0)$, where $l$ is the distance between $\mathrm{M}$ and $\mathrm{M}^{\prime}$. A curvilinear abscissa of $\mathrm{M}^{\prime}$ is equal to $s$, where $s$ is a distance along the path from some arbitrarily chosen point.

If we want to express the position of the point $\mathrm{M}$ not in the classical Cartesian coordinates $(x, y)$ relative to the inertial frame but relative to the given path $P$, we should use some geometric relationships (Mazur, 2004),

$$
\begin{gathered}
\dot{l}=\left(\begin{array}{cc}
-\sin \theta_{r} & \cos \theta_{r}
\end{array}\right)\left(\begin{array}{c}
\dot{x} \\
\dot{y}
\end{array}\right), \\
\dot{s}=\frac{\left(\begin{array}{ll}
\cos \theta_{r} & \sin \theta_{r}
\end{array}\right)}{1-c(s) l}\left(\begin{array}{c}
\dot{x} \\
\dot{y}
\end{array}\right),
\end{gathered}
$$

where $\dot{x}$ and $\dot{y}$ are defined by nonholonomic constraints for each class of wheeled mobile platforms and $\theta_{r}$ is a desired orientation of the platform at the point $M^{\prime}$ on the path. 
The posture of the mobile platform is defined not only by the position of the mass center, but by the orientation, too. For this reason, it is necessary to define the orientation tracking error equal to $\tilde{\theta}=\theta-\theta_{r}$. Moreover, at the point M', the desired orientation of the platform fulfills the following condition (Samson, 1995),

$$
\dot{\theta}_{r}=c(s) \dot{s} .
$$

Then the coordinates

$$
\xi=(l, \tilde{\theta}, s)^{T},
$$

i.e., the Frenet coordinates $(l, s)$ and the orientation tracking error $\tilde{\theta}$, constitute path following errors for a nonholonomic mobile platform. It is worth mentioning that the Frenet parametrization is valid only locally, near the desired path.

If we want to solve the path following problem, it is necessary to express the kinematics of nonholonomic mobile platform in the Frenet coordinates (15) instead of the generalized coordinates $q_{m}$. After using Eqns. (12) and (14), the nonholonomic constraints can be represented by the dynamic driftless system. In our solution to the path following problem for a nonholonomic wheeled mobile platform, we have omitted the differential equation for $\dot{s}$, because it does not matter at which point $s$ of the desired path the mobile platform enters the desired curve $P(s)$, see (Fradkov et al., 1999) for details.

As we have mentioned earlier, out of the Frenet coordinates it is enough to consider only $l$ and $\tilde{\theta}$. Due to the expressions (12) and (14), the Frenet variables for a mobile platform of the $(2,0)$ class described by the nonholonomic constraints

$$
\left(\begin{array}{c}
\dot{x} \\
\dot{y} \\
\dot{\theta}
\end{array}\right)=\left[\begin{array}{cc}
\cos \theta & 0 \\
\sin \theta & 0 \\
0 & 1
\end{array}\right]\left(\begin{array}{l}
v \\
\omega
\end{array}\right)=G\left(q_{m}\right) \eta
$$

can be defined as follows:

$$
\left\{\begin{array}{l}
\dot{l}=v \sin \tilde{\theta} \\
\dot{\tilde{\theta}}=\dot{\theta}-\dot{\theta}_{r}=\omega-v \cos \tilde{\theta} \frac{c(s)}{1-c(s) l}=w
\end{array}\right.
$$

where $w$ is a new control input for the second equation.

In the Samson algorithm, $v$ and $w$ are defined in such a way that $v$ does not converge to zero in time and $w$ depends on $v$ directly. In our discussion, we assume $v(t)=$ const, because we want to preserve a uniform motion along the desired path. Then the Samson kinematic controller generates reference signals equal to

$$
\left\{\begin{array}{l}
v=\text { const } \\
w=-k_{2} l v \frac{\sin \tilde{\theta}}{\tilde{\theta}}-k_{3} \tilde{\theta}, \quad k_{2}, k_{3}>0 .
\end{array}\right.
$$

Proof. For the dynamical system (17) we choose the following Lyapunov-like function:

$$
V_{1}(\xi)=k_{2} \frac{l^{2}}{2}+\frac{\tilde{\theta}^{2}}{2} .
$$

Next we compute a time derivative $\dot{V}_{1}$ along solutions of the closed-loop system (17)-18),

$$
\dot{V}_{1}=k_{2} l i+\tilde{l} \dot{\tilde{\theta}}=-k_{3} \tilde{\theta}^{2} \leq 0 .
$$

It can be observed that $V_{1}$ is decreasing along trajectories of the closed-loop system. This implies that $l$ and $\tilde{\theta}$ stay bounded. Then

$$
\ddot{V}_{1}=-k_{3} \tilde{\theta}\left(-k_{2} l v \frac{\sin \tilde{\theta}}{\tilde{\theta}}-k_{3} \tilde{\theta}\right)
$$

is a sum of bounded components, which is equivalent to the condition that $\dot{V}_{1}$ is uniformly bounded. Next, from the Barbalat lemma, we can conclude the convergence of $\dot{V}_{1}$ to 0 , i.e., $\tilde{\theta} \rightarrow 0$.

Because $\tilde{\theta}=0$ is an asymptotically stable equilibrium point of the system (17)-18, it is easy to show that $\ddot{\tilde{\theta}}$ is bounded, too. This implies that $\dot{\tilde{\theta}}$ has to go to 0 . In the expression (18) describing $\dot{\tilde{\theta}}$, the elements $k_{2}, v$ and $\sin \tilde{\theta} / \tilde{\theta}$ are different from 0 near $\tilde{\theta}=0$, therefore the condition $l \rightarrow 0$ should hold. In this way, we have proven that

$$
(l, \tilde{\theta})=(0,0)
$$

is an asymptotically stable equilibrium point of the control system considered. This completes the proof of the convergence of the mobile platform of a $(2,0)$ class to a desired path.

The velocities $\eta_{r}$ coming from the Samson kinematic algorithm, which are solutions to pure mathematical constraints (ideal case), are equal to

$$
\left\{\begin{array}{l}
\eta_{1 r}=v=\text { const } \\
\eta_{2 r}=\omega=-k_{2} l v \frac{\sin \tilde{\theta}}{\tilde{\theta}}-k_{3} \tilde{\theta}+v \cos \tilde{\theta} \frac{c(s)}{1-c(s) l} .
\end{array}\right.
$$

4.1.2. Path following problem for a planar nonholonomic manipulator. In the problem of path following for a planar manipulator located on the $X Z$ plane with nonholonomic gears, the Frenet parametrization presented in Section 4.1.1 can be evoked once again: the role of the point $\mathrm{M}$ in Fig. 5 is played by a point at the end of a gripper, whereas the orientation of the end-effector $\theta_{m}$ is a rotation angle of the frame associated with the gripper around the $-Y_{b}$ axis, located in the base of the manipulator. The orientation of the end-effector in the planar nonholonomic $n$-pendulum is then equal to

$$
\theta_{m}=\sum_{i=1}^{n} \theta_{i}
$$


In the analysed planar nonholonomic manipulator lying in the $X Z$-plane, relationships between the velocity of the working point $\mathrm{M}$ expressed in the Cartesian and curvilinear coordinates have the form

$$
\begin{aligned}
\dot{l}_{m} & =\left(\begin{array}{ll}
-\sin \theta_{r m} & \cos \theta_{r m}
\end{array}\right)\left(\begin{array}{c}
\dot{x} \\
\dot{z}
\end{array}\right), \\
\dot{s} & =\frac{\left(\begin{array}{cc}
\cos \theta_{r m} & \sin \theta_{r m}
\end{array}\right)}{1-c(s) l_{m}}\left(\begin{array}{c}
\dot{x} \\
\dot{z}
\end{array}\right),
\end{aligned}
$$

where $l_{m}$ denotes the distance between the point $\mathrm{M}$ and the path $\Pi(s)$, and $\theta_{r m}$ is the orientation of the Frenet frame in the point M'. Subscripts were introduced to distinguish the Frenet variables for both subsystems of the $(n h, n h)$ mobile manipulator.

Coordinates of the end-effector in the $n$-pendulum relative to its base are equal to

$$
\left\{\begin{array}{l}
x=\sum_{i=1}^{n} l_{i} \cos \left(\sum_{j=1}^{i} \theta_{j}\right), \\
z=\sum_{i=1}^{n} l_{i} \sin \left(\sum_{j=1}^{i} \theta_{j}\right) .
\end{array}\right.
$$

After substituting time derivatives of the variables (25) into the expressions (23), we obtain the following equations:

$$
\begin{aligned}
\dot{l}_{m}= & \sum_{i=1}^{n} \cos \left(\theta_{r m}-\sum_{j=1}^{i} \theta_{j}\right) l_{i} \sum_{k=1}^{i} \dot{\theta}_{k}, \\
\dot{\tilde{\theta}}_{m}= & \dot{\theta}_{m}-c(s) \dot{s}=\sum_{i=1}^{n} \dot{\theta}_{i}-\frac{c(s)}{1-c(s) l_{m}} \\
& \cdot \sum_{i=1}^{n} \sin \left(\theta_{r m}-\sum_{j=1}^{i} \theta_{j}\right) l_{i} \sum_{k=1}^{i} \dot{\theta}_{k} .
\end{aligned}
$$

Using the kinematics of the nonholonomic manipulator given by (3) and (4), Eqns. (26) and (27) can be expressed in matrix form as follows:

$$
\begin{aligned}
\dot{\xi}_{m} & =\left(\begin{array}{c}
\dot{l}_{m} \\
\dot{\tilde{\theta}}_{m}
\end{array}\right)=H\left(q_{r}, \xi_{m}\right)\left(\begin{array}{c}
\dot{\theta}_{1} \\
\dot{\theta}_{2} \\
\vdots \\
\dot{\theta}_{n}
\end{array}\right) \\
& =H\left(q_{r}, \xi_{m}\right) G_{2}\left(q_{r}\right) u=K_{l}\left(q_{r}, \xi_{m}\right) u .
\end{aligned}
$$

The matrix $K_{l}\left(q_{r}, \xi_{m}\right)$ fulfills a regularity condition (i.e., it is invertible) if some configurations, which imply matrix singularity, are excluded from a set of possibly achieved poses of the nonholonomic manipulator. Singular configurations for a nonholonomic 3-pendulum are calculated in Section 6.1.

For the regular matrix $K_{l}$, the following reference control signals guaranteeing the convergence of tracking errors to zero in the ideal case (i.e., for pure kinematic constraints without dynamics), can be proposed:

$$
u_{r}=-K_{l}^{-1}\left(q_{r}, \xi_{m}\right) \Lambda \xi_{m}, \quad \Lambda=\Lambda^{T}>0 .
$$

It is easy to observe that the system (28) with closed-loop of the feedback signal (29) has the form

$$
\dot{\xi}_{m}+\Lambda \xi_{m}=0
$$

i.e., it is asymptotically stable.

4.2. Path following problem for a holonomic subsystem. The desired path of the manipulator can be specified by the geometric curve $\Pi(s)$ for the end-effector. Such the path given in the $\mathbb{R}^{p}$ local workspace is defined relative to the manipulator local base's frame $X_{b} Y_{b}$, see Fig. 1, where $\rho \leq p$. The goal of the control process is to move the end-effector along the path $\Pi(s)$, and the vector of tracking errors has to be asymptotically convergent, i.e.,

$$
e_{p}=k\left(q_{r}\right)-\Pi(s) \longrightarrow 0 .
$$

Similarly to the Frenet parametrization, $s$ is a current parameter describing the path, i.e., its curvilinear length. $k\left(q_{r}\right)$ are the kinematics of the manipulator, which describe position of the end-effector and sometimes its orientation, if some relationship between the orientation and the parameter $s$ holds (i.e., orientation depends on the parameter $s$ ). We will assume that the mapping $\partial k / \partial q_{r}=$ $J$, which is, in fact, a Jacobi matrix for the manipulator, has always full rank $\rho$ (manipulator avoids singular configurations). Moreover, we make the assumption that first and second derivatives of the path $\Pi(s)$ exist and they are bounded.

Due to (Galicki, 2006), where the idea of path following for redundant manipulators was formulated, we assume that at the initial moment, for which $s(0)=0$ holds, the manipulator's end-effector is located on the path, i.e.,

$$
k\left(q_{r}(0)\right)=\Pi(0),
$$

and that at the initial and the final moment the end-effector does not move,

$$
\dot{s}(0)=\dot{s}(T)=0, \quad T \longrightarrow \infty .
$$

Moreover, because the workspace of the robotic arm is not very big and the end-effector should move along the path with stopping at the end, we expect that the length of the path is limited to the value $s_{\max }$. This implies that the next error (curvilinear distance from the end point of the path) should be defined,

$$
e_{s}=s-s_{\max }
$$




\section{Path following for a nonholonomic mobile manipulator of the $(n h, h)$ type}

To obtain path following for a mobile manipulator of the $(n h, h)$ type, two expressions have to be defined:

- $\dot{s}(t)$ — path parametrization for the manipulator,

- $\tau$-control algorithm for the dynamics designed for the full $(n h, h)$ mobile manipulator.

5.1. Path parametrization for the arm. The path parametrization $s=s(t)$ can be obtained from the scalar differential equation (Galicki, 2006),

$$
\begin{aligned}
\ddot{s} & =-K_{d} \dot{s}-K_{m} e_{s}\left(\frac{1}{2} \frac{\partial \gamma}{\partial s} e_{s}+\gamma\right)+2 K_{2} e_{p}^{T} F, \\
F & =\frac{\partial \Pi}{\partial s},
\end{aligned}
$$

where $K_{d}, K_{m}, K_{2}>0$ are some regulation parameters and $\gamma \in C^{2}$ is assumed to be a strictly positive function of $s$ (with the first and second derivatives bounded), which cannot fulfill the differential equation

$$
\frac{1}{2} \frac{\partial \gamma}{\partial s} e_{s}+\gamma=0
$$

This equation has no special physical meaning; it is similar to a specific restriction on regulation parameters. On the other hand, it is very easy to fulfill this requirement. The function $\gamma$ can be equal to 1 , but if it is in a more general form, then it is possible to achieve a more flexible behavior of $e_{s}$ near the endpoint of the path.

5.2. Dynamic control. We will formulate the main result as a theorem.

Theorem 1. Let us consider the model of a mobile manipulator (7) with the nonholonomic constraints (2). We assume that the reference kinematic control $\eta_{r}(t)$ computed due to the Samson algorithm (22) solves the path following problem for the mobile platform.

Then we propose a dynamic control law,

$$
\begin{aligned}
\tau= & \left(B^{*}\right)^{-1}\left\{Q^{*}\left(\begin{array}{c}
\dot{\eta}_{r} \\
-\dot{J}^{T} e_{p}-J^{T} J \dot{q}_{r}+J^{T} F \dot{s}
\end{array}\right)\right. \\
& \left.+C^{*}\left(\begin{array}{c}
\eta_{r} \\
-J^{T} e_{p}
\end{array}\right)+D^{*}-C_{K} E_{v}-K E_{v}\right\}
\end{aligned}
$$

where $F$ is defined by (31) and the symbols have the following meaning:

$$
\begin{aligned}
E_{v} & =\left(\begin{array}{c}
e_{\eta} \\
\dot{q}_{r}+J^{T} e_{p}
\end{array}\right)=\left(\begin{array}{c}
\eta-\eta_{r} \\
\dot{q}_{r}+J^{T} e_{p}
\end{array}\right), \\
K & =\left[\begin{array}{cc}
K_{11} & 0 \\
0 & K_{12}
\end{array}\right], \\
K_{1 i} & =\operatorname{diag}\left\{K_{i}\right\}, \quad K_{i}>0, \quad i=1,2,
\end{aligned}
$$

which preserves asymptotic convergence for full kinematic and dynamic coordinates of the mobile manipulator to their desired values. This implies path following for each subsystem of the mobile manipulator.

Proof. To prove proper behavior of the presented dynamic control algorithm, it is necessary to take under consideration not only dynamic algorithm given by (32), but the kinematic controller, too.

The closed-loop system (7) and (32) is described by the error equation

$$
Q^{*} \dot{E}_{v}=-K E_{v}-\left(C^{*}+C_{K}\right) E_{v}
$$

In order to prove the convergence of trajectories of both subsystems of the mobile manipulator to the desired paths, we choose the following Lyapunov-like function:

$$
\begin{aligned}
W\left(\xi, E_{v}, e_{s}, \dot{s}\right)= & V_{1}(\xi)+\frac{1}{2} E_{v}^{T} Q^{*} E_{v}+\frac{1}{2} \dot{s}^{2} \\
& +\frac{1}{2} \gamma K_{m} e_{s}^{2}+K_{2} e_{p}^{T} e_{p},
\end{aligned}
$$

where $V_{1}(\xi)$ is the Lyapunov function (19) for the kinematics (17) expressed in the Frenet variables (transformed to the path following problem). Now we calculate the time derivative of $W$ as follows:

$$
\begin{aligned}
\dot{W}= & \dot{V}_{1}+E_{v}^{T} Q^{*} \dot{E}_{v}+\frac{1}{2} E_{v}^{T} \dot{Q}^{*} E_{v}+\dot{s} \ddot{s} \\
& +\left(\frac{1}{2} \frac{\partial \gamma}{\partial s} e_{s}^{2}+\gamma e_{s}\right) K_{m} \dot{s}+2 K_{2} e_{p}^{T} \dot{e}_{p}
\end{aligned}
$$

Before we start to evaluate $\dot{W}$ along trajectories of the closed-loop system (17), 22), (31) and (33), it is necessary to describe the influence of the additional errors $e_{\eta}$, defined in Theorem 1, which come from the dynamic control level and disturb solutions to the kinematic equations (22). We will treat $\eta_{r}$ as kinematic control signals in the ideal case (i.e., without dynamics), and then, as kinematic control for a real case (with dynamics), instead $\eta_{i r}$ defined by (22), we should take disturbed controls as follows:

$$
v=\text { const }+e_{\eta 1},
$$

$$
\begin{aligned}
\omega= & -k_{2} l v \frac{\sin \tilde{\theta}}{\tilde{\theta}}-k_{3} \tilde{\theta} \\
& +v \cos \tilde{\theta} \frac{c(s)}{1-c(s) l}+e_{\eta 2} .
\end{aligned}
$$

Now we evaluate the time derivative of $W$ along trajectories of the closed-loop system (17)- 36 in the fol- 
lowing way:

$$
\begin{aligned}
\dot{W}= & -k_{3} \tilde{\theta}^{2}+\tilde{\theta} e_{\eta 2}-e_{\eta}^{T} K_{11} e_{\eta}-K_{2} \dot{q}_{r}^{T} \dot{q}_{r}-K_{d} \dot{s}^{2} \\
& -K_{2} e_{p}^{T} J J^{T} e_{p} \\
= & -\left(k_{3}-\frac{1}{2}\right) \tilde{\theta}^{2}-\frac{1}{2}\left(\tilde{\theta}-e_{\eta 2}\right)^{2}-K_{1} e_{\eta 1}^{2}-K_{d} \dot{s}^{2} \\
& -\left(K_{1}-\frac{1}{2}\right) e_{\eta 2}^{2}-K_{2} \dot{q}_{r}^{T} \dot{q}_{r}-K_{2} e_{p}^{T} J J^{T} e_{p} \\
\leq & -\left(k_{3}-\frac{1}{2}\right) \tilde{\theta}^{2}-K_{1} e_{\eta 1}^{2}-\left(K_{1}-\frac{1}{2}\right) e_{\eta 2}^{2} \\
& -K_{2} \dot{q}_{r}^{T} \dot{q}_{r}-K_{d} \dot{s}^{2}-K_{2} e_{p}^{T} J J^{T} e_{p} \\
\leq & 0
\end{aligned}
$$

It is easy to see that the Lyapunov function $W$ is decreasing along any trajectory of the closed-loop system, if control parameters are greater than properly chosen numbers, i.e., $K_{1}, k_{3}>1 / 2$.

The matrix $J J^{T}$ is a matrix of manipulability of the rigid manipulator and it is always positive definite as a consequence of the assumption about avoiding singularities by the manipulator. From LaSalle's invariance principle, we conclude that

$$
\left(\tilde{\theta}, e_{\eta 1}, e_{\eta 2}, \dot{q}_{r}, \dot{s}, e_{p}\right)=(0,0,0,0,0,0)
$$

is an asymptotically stable equilibrium point. The convergence of $l$ is a consequence of the deliberations and arguments presented in Section 4.1.1

Now we want to prove the convergence of $e_{s}$ to zero. The function $W$ is decreasing along trajectories of the closed-loop system, therefore all signals defining this function (i.e., $\xi, E_{v}, \dot{s}, e_{s}$ and $e_{p}$ ) are bounded. Using properties of the functions $\gamma$ and $\Pi$ and the boundedness of the above-mentioned variables, it is easy to prove that $\ddot{s}$, which is a sum of products of bounded variables, is bounded and, consequently, so is

$$
\begin{aligned}
s^{(3)}= & -K_{d} \ddot{s}-2 K_{m} e_{s} \dot{s} \frac{\partial \gamma}{\partial s}-\frac{1}{2} K_{m} e_{s}^{2} \frac{\partial^{2} \gamma}{\partial s^{2}} \dot{s}-K_{m} \dot{s} \gamma \\
& +2 K_{2}\left(\dot{q}_{r}^{T} J^{T}-F^{T} \dot{s}\right) F+2 K_{2} e_{p}^{T} \frac{\partial^{2} \Pi}{\partial s^{2}} \dot{s} .
\end{aligned}
$$

Applying above arguments to the function $\dot{s}$, due to the Barbalat lemma, we conclude that $\ddot{s}(T) \rightarrow 0$ for $T \rightarrow$ $\infty$. From the equation of path parametrization (31), we can see that the following equation is fulfilled:

$$
\ddot{s}(\infty)=0 \longrightarrow \frac{1}{2} \frac{\partial \gamma}{\partial s} e_{s}^{2}(\infty)+\gamma e_{s}(\infty)=0 .
$$

This means that either $e_{s}(\infty)=0$ or $\frac{1}{2} \frac{\partial \gamma}{\partial s} e_{s}(\infty)+\gamma=$ 0 . However, from the properties of the $\gamma(s)$ function, we know that the second option does not hold. Thus, $e_{s}(\infty)=0$. This completes the proof.
5.3. Simulation study for an $(n h, h)$ mobile manipulator. The simulations were run with the MATLAB package and the SIMULINK toolbox. As an object of simulations we chose a rigid RTR manipulator mounted on the unicycle, depicted in Fig. 1. The kinematics of the platform are given by (16). The dynamics are omitted in the text for the sake of conciseness, but they can be found in (Mazur, 2000).

The goal of the simulations was to investigate the behavior of a mobile manipulator with the dynamic controller (31), (32) and the kinematic controller (22). The desired path for the manipulator (straight line) was selected as

$$
\begin{aligned}
\Pi_{1}(s) & =0.4[\mathrm{~m}], \\
\Pi_{2}(s) & =0.866 s[\mathrm{~m}], \\
\Pi_{3}(s) & =0.5 s+0.5[\mathrm{~m}], \\
s_{\max } & =0.2[\mathrm{~m}], \\
\gamma(s) & =1,
\end{aligned}
$$

and the desired path for the mobile platform was the other straight line,

$$
x(s)=\frac{\sqrt{2}}{2} s[\mathrm{~m}], \quad y(s)=\frac{\sqrt{2}}{2}[\mathrm{~m}] .
$$

The initial configuration of the manipulator was equal to $\left(\theta_{1}, \theta_{2}, \theta_{3}\right)(0)=(0,0.6732,-\pi / 3)$, and the initial posture of the platform was selected as $(x, y, \theta)(0)=$ $(0,2,3 \pi / 4)$.

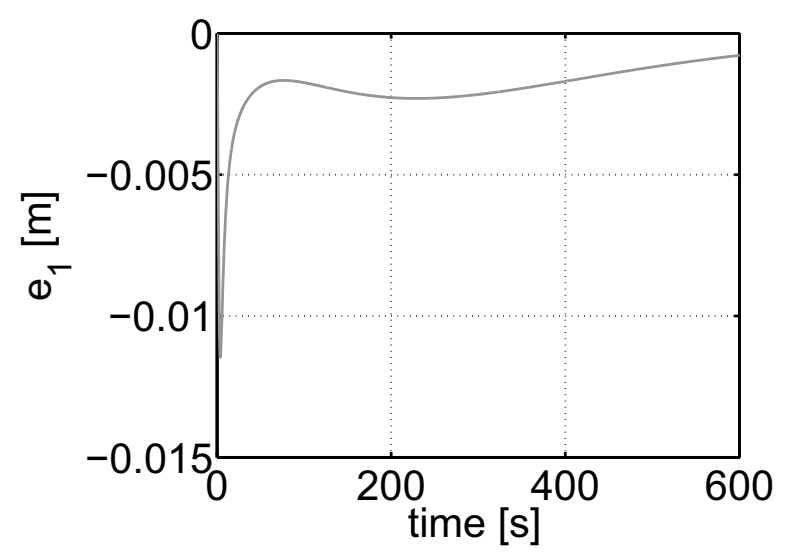

Fig. 6. Error of the $x$ coordinate for the RTR manipulator.

The tracking of the desired path by the end-effector of the RTR manipulator is depicted in Figs. 6, 8 . The tracking of the desired path for the mobile platform is presented in Figs. 9 911 , The parameters of the dynamic controller were equal to $K_{d}=K_{m}=1, K_{1}=1$ and $K_{2}=0.01$. In turn, the parameters of the kinematic Samson algorithm were selected as $v=1, k_{2}=0.1$ and $k_{3}=1$. 


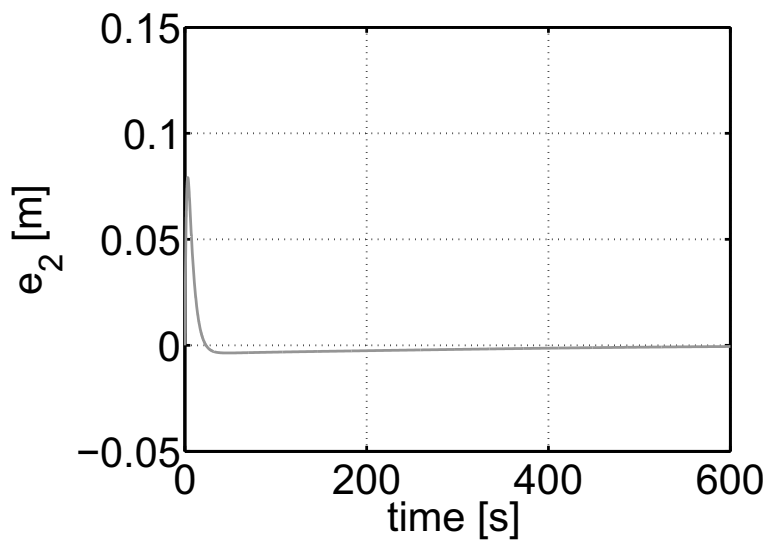

Fig. 7. Error of the $y$ coordinate for the RTR manipulator.

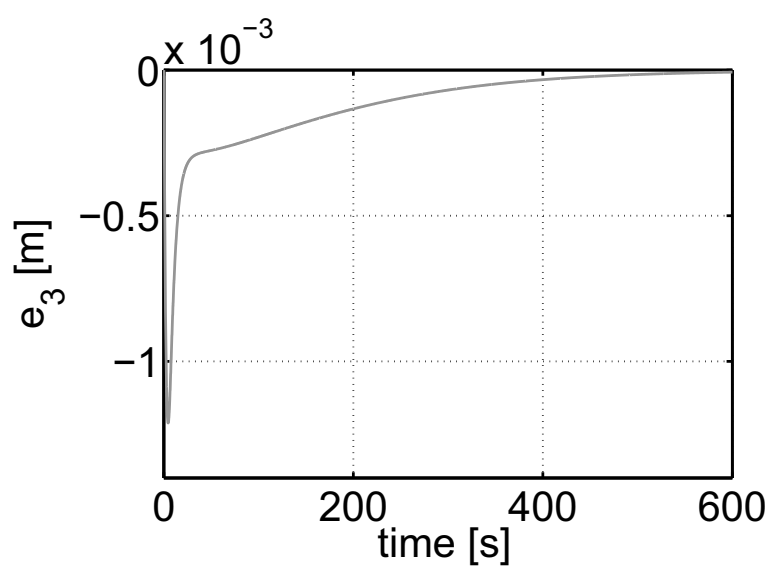

Fig. 8. Error of $z$ coordinate for the RTR manipulator.

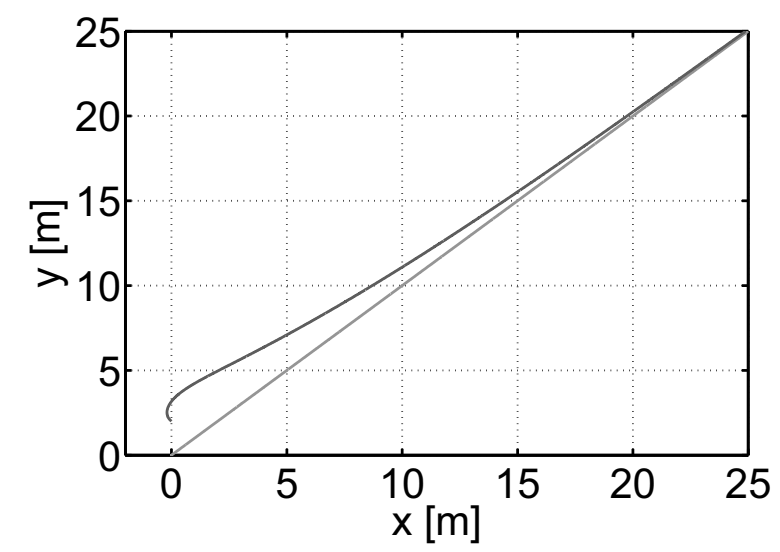

Fig. 9. Path tracking for the mobile platform-XY plot.

It is easy to observe that the convergence of coordinate errors, especially for the $x$ and $z$ coordinates, is very slow. The convergence ratio depends on the gains $K_{1}$, $K_{2}$ in the dynamic controller in such a way that bigger values of regulation parameters imply faster convergence.

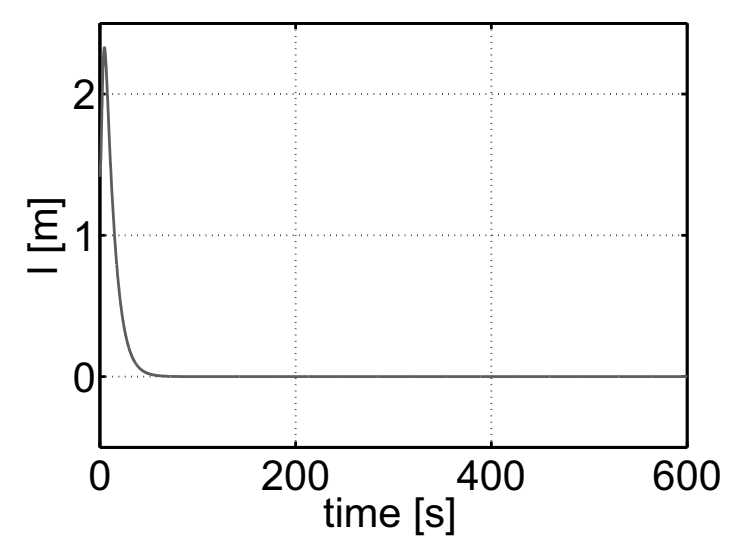

Fig. 10. Path tracking for the mobile platform—distance error $l$.

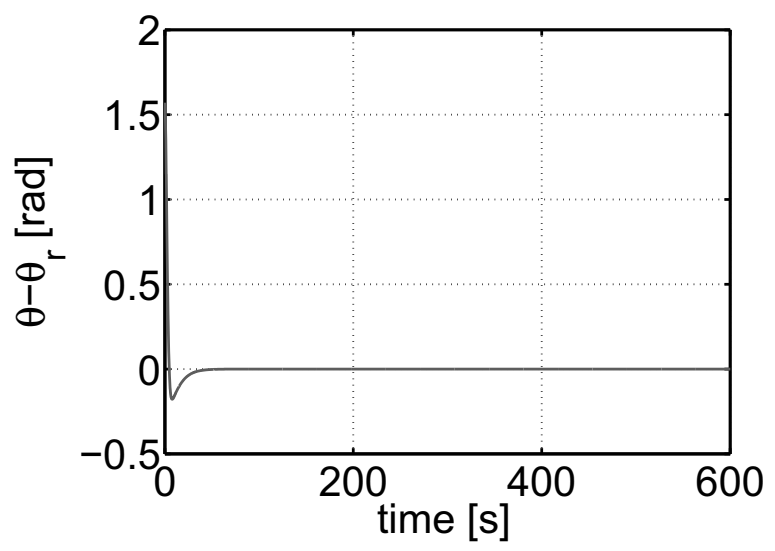

Fig. 11. Path tracking for the mobile platform—orientation er$\operatorname{ror} \tilde{\theta}$.

On the other side, the performance of the dynamic control algorithm depends on the specific construction of the holonomic rigid manipulator. The RTR manipulator can move in the $\mathbb{R}^{3}$ space, and therefore its displacement is more difficult to control than in the case of any planar manipulator.

From the plots presented in Figs. 9 11 we can see that in the Samson algorithm fast convergence is easy to notice.

\section{Path following for a nonholonomic mobile manipulator of the $(n h, n h)$ type}

Similarly to the $(n h, h)$ mobile manipulator, we will formulate the main result as a theorem.

Theorem 2. Let us consider the model of a mobile manipulator (10) with the nonholonomic constraints (9). We assume that the reference kinematic controls $\zeta_{r}(t)=$ $\left(\eta_{r}^{T}(t), u_{r}^{T}(t)\right)$ solve the path following problem for both nonholonomic subsystems, where $\eta_{r}(t)$ was computed due 
to the Samson algorithm (22) for the mobile platform and $u_{r}(t)$ for the nonholonomic manipulator is given by (29).

Then we propose a dynamic control law,

$$
\tau=\left(B^{*}\right)^{-1}\left\{Q^{*}\left(\begin{array}{c}
\dot{\eta}_{r} \\
\dot{u}_{r}
\end{array}\right)+C^{*}\left(\begin{array}{c}
\eta_{r} \\
u_{r}
\end{array}\right)+D^{*}-\left(K+C_{K}\right) E_{\zeta}\right\},
$$

with the symbols defined as follows:

$$
E_{\zeta}=\left(\begin{array}{c}
e_{\eta} \\
e_{u}
\end{array}\right)=\left(\begin{array}{l}
\eta-\eta_{r} \\
u-u_{r}
\end{array}\right), K=\left[\begin{array}{cc}
K_{1} I_{2} & 0 \\
0 & K_{2} I_{2}
\end{array}\right],
$$

and $K_{1}, K_{2}>0$, which preserves asymptotic convergence for full kinematic and dynamic coordinates of the $(n h, n h)$ mobile manipulator to their desired values.

Proof. In order to prove the convergence of trajectories of both subsystems of the mobile manipulator to the desired paths, we choose the following Lyapunov-like function:

$$
V\left(\xi, \xi_{m}, E_{\zeta}\right)=k_{2} \frac{l^{2}}{2}+\frac{\tilde{\theta}^{2}}{2}+\xi_{m}^{T} \xi_{m}+\frac{1}{2} E_{\zeta}^{T} Q^{*} E_{\zeta}
$$

Similarly to the case of the $(n h, h)$ mobile manipulator, it is necessary to take into consideration the influence of the additional errors $E_{\zeta}$, which come from the dynamic control level and disturb kinematic reference signals. We will treat $\zeta_{r}=\left(\eta_{r}, u_{r}\right)$ as kinematic control signals in the ideal case. Then, as kinematic control for a real case, instead of $\eta_{i r}$ we should take the disturbed controls (35), (36), and instead of $u_{r}$ it is necessary to take the disturbed signals

$$
u=-K_{l}^{-1}\left(q_{r}, \xi_{m}\right) \Lambda \xi_{m}+e_{u} .
$$

Putting to the expression for the time derivative of $V$

$$
\dot{V}=k_{2} l \dot{l}+\tilde{\theta} \dot{\tilde{\theta}}+\xi_{m}^{T} \dot{\xi}_{m}+\frac{1}{2} E_{\zeta}^{T} \dot{Q}^{*} E_{\zeta}+E_{\zeta}^{T} Q^{*} \dot{E}_{\zeta}
$$

the equation of the closed-loop system (10), (38),

$$
Q^{*} \dot{E}_{\zeta}+\left(C^{*}+C_{K}\right) E_{\zeta}+K E_{\zeta}=0,
$$

and the equation of the disturbed kinematic controls (35), (36) and (40), we obtain

$$
\begin{aligned}
\dot{V}= & -k_{3} \tilde{\theta}^{2}+\tilde{\theta} e_{\eta 2}-\xi_{m}^{T} \Lambda \xi_{m}+\xi_{m}^{T} K_{l} e_{u}-E_{\zeta}^{T} K E_{\zeta} \\
= & -k_{3} \tilde{\theta}^{2}+\tilde{\theta} e_{\eta 2}-\xi_{m}^{T} \Lambda \xi_{m}+\xi_{m}^{T} K_{l} e_{u}-e_{\eta}^{T} K_{1} e_{\eta} \\
& -e_{u}^{T} K_{2} e_{u} \\
= & -\left(k_{3}-\frac{1}{2}\right) \tilde{\theta}^{2}-\frac{1}{2}\left(\tilde{\theta}-e_{\eta 2}\right)^{2}-\left(K_{1}-\frac{1}{2}\right) e_{\eta 2}^{2} \\
& -K_{1} e_{\eta 1}^{2}-\xi_{m}^{T}\left(\Lambda-\frac{I_{2}}{2}\right) \xi_{m}+\frac{1}{2} e_{u}^{T} K_{l}^{T} K_{l} e_{u} \\
& -\frac{1}{2}\left(\xi_{m}-K_{l} e_{u}\right)^{T}\left(\xi_{m}-K_{l} e_{u}\right)-e_{u}^{T} K_{2} e_{u} .
\end{aligned}
$$

From the definition of the Frenet variables $\xi_{m}$ it is known that a norm of the matrix $K_{l}\left(q_{r}, \xi_{m}\right)$ is bounded and there holds

$$
\left\|K_{l}\left(q_{r}, \xi_{m}\right)\right\| \leq K_{\max }<\infty .
$$

Then the time derivative of the Lyapunov function can be evaluated as follows:

$$
\begin{aligned}
\dot{V}= & -\left(k_{3}-\frac{1}{2}\right) \tilde{\theta}^{2}-\frac{1}{2}\left(\tilde{\theta}-e_{\eta 2}\right)^{2}-\left(K_{1}-\frac{1}{2}\right) e_{\eta 2}^{2} \\
& -K_{1} e_{\eta 1}^{2}-\xi_{m}^{T}\left(\Lambda-\frac{I_{2}}{2}\right) \xi_{m}+\frac{1}{2} e_{u}^{T} K_{l}^{T} K_{l} e_{u} \\
& -\frac{1}{2}\left(\xi_{m}-K_{l} e_{u}\right)^{T}\left(\xi_{m}-K_{l} e_{u}\right)-e_{u}^{T} K_{2} e_{u} \\
\leq & -\left(k_{3}-\frac{1}{2}\right) \tilde{\theta}^{2}-\left(K_{1}-\frac{1}{2}\right) e_{\eta 2}^{2}-K_{1} e_{\eta 1}^{2} \\
& -\xi_{m}^{T}\left(\Lambda-\frac{I_{2}}{2}\right) \xi_{m}-e_{u}^{T}\left(K_{2}-\frac{K_{\max }^{2}}{2}\right) e_{u} \\
\leq & -W\left(\tilde{\theta}, E_{\zeta}, \xi_{m}\right) \leq 0 .
\end{aligned}
$$

It can be seen that if regulation parameters are properly chosen, i.e.,

$$
k_{3}>\frac{1}{2}, K_{1}>\frac{1}{2}, \Lambda>\frac{I_{2}}{2}, K_{2}>\frac{K_{\max }^{2}}{2},
$$

then the above evaluation fulfills the assumptions of the LaSalle theorem (Krstić et al., 1995). This means that the point

$$
\left(\tilde{\theta}, e_{\eta}, e_{u}, \xi\right)=(0,0,0,0)
$$

is a locally asymptotically stable equilibrium point of trajectories of the mobile manipulator with the control signals (35), (36) and (38). This completes the proof.

6.1. Simulation study for an $(n h, n h)$ mobile manipulator. As an object of simulations we chose a nonholonomic vertical 3-pendulum mounted on the unicycle. The nonholonomic constraints for the mobile platform are given by (16) and for the nonholonomic robotic arm they are defined as (3)-(4). The dynamics of the mobile manipulator considered are omitted for the sake of clarity, but they can be found in (Mazur, 2000).

For a nonholonomic 3-pendulum, the matrix $K_{l}\left(q_{r}, \xi_{m}\right)$ has the form

$$
K_{l}\left(q_{r}, \xi_{m}\right)=\left[\begin{array}{ll}
K_{l 11} & K_{l 12} \\
K_{l 21} & K_{l 22}
\end{array}\right]
$$

with the elements defined below:

$$
\begin{aligned}
K_{l 11}= & \sum_{i=1}^{3} l_{i} \cos \left(\theta_{r m}-\sum_{j=1}^{i} \theta_{i}\right), \\
K_{l 12}= & a_{2} s_{1} \sum_{i=2}^{3} l_{i} \cos \left(\theta_{r m}-\sum_{j=1}^{i} \theta_{i}\right) \\
& +a_{3} s_{2} c_{1} l_{3} \cos \left(\theta_{r m}-\sum_{j=1}^{3} \theta_{i}\right), \\
K_{l 21}= & 1-\frac{c(s)}{1-c(s) l_{m}} \sum_{i=1}^{3} l_{i} \sin \left(\theta_{r m}-\sum_{j=1}^{i} \theta_{i}\right),
\end{aligned}
$$




$$
\begin{aligned}
K_{l 22}= & a_{2} s_{1}\left[1-\frac{c(s)}{1-c(s) l_{m}} \sum_{i=2}^{3} l_{i} \sin \left(\theta_{r m}-\sum_{j=1}^{i} \theta_{i}\right)\right] \\
& +a_{3} s_{2} c_{1}\left[1-\frac{c(s)}{1-c(s) l_{m}} l_{3} \sin \left(\theta_{r m}-\sum_{j=1}^{3} \theta_{i}\right)\right] .
\end{aligned}
$$

It is worth mentioning that the Frenet transformation is valid only locally, i.e., $l_{m}(0)<r_{\min }$, where $r_{\min }$ is an inversion of the maximal curvature $c_{\max }$ of the manipulator path $\Pi(s)$, and therefore nominators of all fractions are well defined. In turn, the nonholonomic planar 3-pendulum cannot achieve angles equal to $\theta_{1}, \theta_{2}=$ $0, \pm \pi$. Moreover, singularities in the $K_{l}$ matrix occur for $\cos \left(\theta_{r m}-\theta_{1}\right)=\cos \left(\theta_{r m}-\theta_{1}-\theta_{2}\right)=\cos \left(\theta_{r m}-\theta_{1}-\right.$ $\left.\theta_{2}-\theta_{3}\right)=0$.

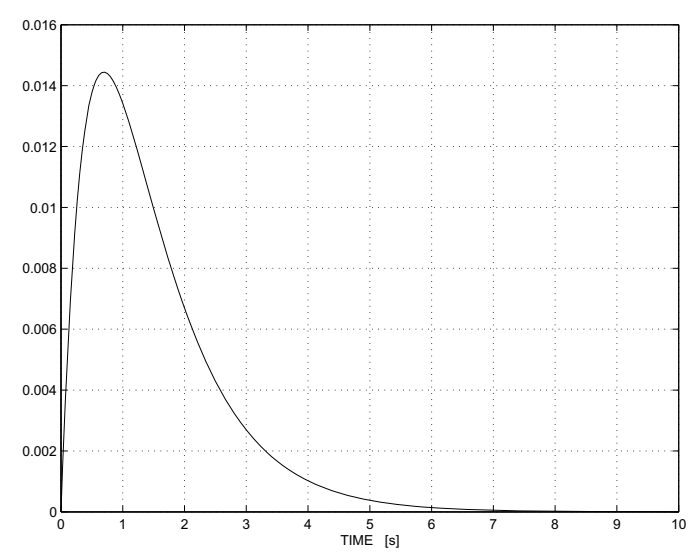

Fig. 12. Path tracking for the 3-pendulum: an error of the $x$ coordinate.

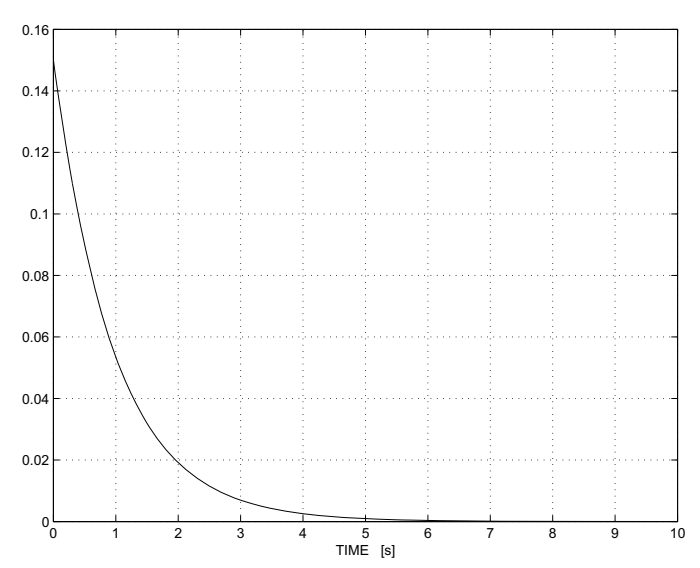

Fig. 13. Path tracking for the 3-pendulum: an error of the $z$ coordinate.

The desired path for the manipulator (circle) was selected as

$$
\begin{aligned}
& \Pi_{1}(s)=0.25 \cos 4 s+1[\mathrm{~m}], \\
& \Pi_{2}(s)=-0.25 \sin 4 s+0.6[\mathrm{~m}],
\end{aligned}
$$

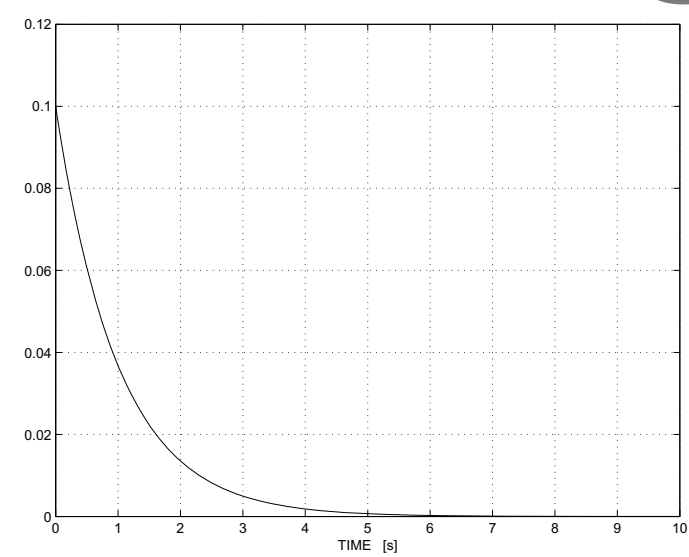

Fig. 14. Path tracking for the 3-pendulum: an error of orientation $\tilde{\theta}$.

and the desired path for the mobile platform was the same straight line as for the $(n h, h)$ mobile manipulator.

The initial configuration of the manipulator was equal to $\left(\theta_{1}, \theta_{2}, \theta_{3}\right)(0)=(0, \pi / 2,-\pi / 2+0.1)$ and the initial posture of the platform was selected as $(x, y, \theta)(0)=$ $(0,2,3 \pi / 4)$.

The tracking of the desired path by the end-effector of the 3-pendulum is depicted in Figs. 12 14 The parameters of the dynamical controller were equal to $K_{1}=$ $K_{2}=100$. In turn, the parameters of the kinematic Samson algorithm for the platform were selected as $v=1$, $k_{2}=0.1$ and $k_{3}=1$. The matrix $\Lambda$ in the kinematic algorithm dedicated the nonholonomic manipulator was a diagonal matrix with 2 on the diagonal.

From the plots presented in Figs. 12 14, it is easy to observe that the convergence of the coordinate errors of $x$ and $z$ is rather fast. Such good convergence is a result of big values for dynamic controller gains. On the other hand, big gains in the dynamic control algorithm are necessary, because the gear ratios $a_{2}=0.1$ and $a_{3}=0.2$ appearing in the equations of nonholonomic constraints for a planar 3-pendulum are small.

\section{Conclusions}

The most important result obtained in this work shows clearly that the path following problem for nonholonomic mobile manipulators can be solved using the control laws introduced in this paper. These dynamic control algorithms are locally asymptotically stable. Simulation results confirm good behavior of the mobile manipulatornonholonomic platform moves along a desired contour without the slippage of its wheels and the onboard manipulator executes some tasks defined relative to the platform, which makes it possible to unload a payload during the transportation. Moreover, in the paper, the kinematic controller guaranteeing path following for a nonholonomic 
manipulator was designed.

It is worth emphasising that the presented dynamical controllers can be applied only to a mobile manipulator with full knowledge about its dynamics. However, for holonomic manipulators, it has been shown in the literature that it is possible to move the robotic arm along the prescribed path without any knowledge about its dynamics. For this reason, future works could focus on extending the existing controllers to adaptive versions, i.e., for a nonholonomic mobile manipulator with parametric uncertainty in the dynamics.

\section{References}

Canudas de Wit, C., Siciliano, B. and Bastin, G. (1996). Theory of Robot Control, Springer-Verlag, London.

Chung, J., Velinsky, S. and Hess, R. (1998). Interaction control of a redundant mobile manipulator, International Journal of Robotics Research 17(12): 1302-1309.

D’Andréa-Novel, B., Bastin, G. and Campion, G. (1991). Modelling and control of nonholonomic wheeled mobile robots, Proceedings of the IEEE International Conference on Robotics and Automation, Sacramento, CA, USA, pp. 1130-1135.

Dong, W. (2002). On trajectory and force tracking control of constrained mobile manipulators with parameter uncertainty, Automatica 38(9): 1475-1484.

Dulęba, I. (2000). Modeling and control of mobile manipulators, Proceedings of the 6th IFAC Symposium on Robot Control, SYROCO'00, Vienna, Austria, pp. 687-692.

Fradkov, A., Miroshnik, I. and Nikiforov, V. (1999). Nonlinear and Adaptive Control of Complex Systems, Kluwer Academic Publishers, Dordrecht.

Galicki, M. (2006). Adaptive control of kinematically redundant manipulator, Lecture Notes in Control and Information Sciences (335): 129-139.

Hatano, M. and Obara, H. (2003). Stability evaluation for mobile manipulators using criteria based on reaction, Proceedings of the SICE Annual Conference, Fukui, Japan, pp. 2050-2055.

Huang, Q., Sugano, S. and Tanie, K. (1998). Motion planning for a mobile manipulator considering stability and task constraints, Proceedings of the IEEE International Conference on Robotics and Automation, Leuven, Belgium, pp. 2192-2198

Khatib, O. (1999). Mobile manipulation: The robotic assistant, Journal on Robotics and Autononous Systems 26: 175-183.

Krstić, M., Kanellakopoulos, I. and Kokotović, P. (1995). Nonlinear and Adaptive Control Design, J. Wiley and Sons, New York, NY.

Li, Z., Ge, S. and Ming, A. (2007). Adaptive robust motion/force control of holonomic-constrained nonholonomic mobile manipulator, IEEE Transactions on System, Man and Cybernetics, Part B: Cybernetics 37(3): 607-617.
Mazur, A. (2000). Comparative study of control algorithms for nonholonomic mobile manipulators, Technical Report SPR 38/00, Institute of Engineering Cybernetics, Wrocław University of Technology, http://sequoia.ict.pwr.wroc.pl/ alicja (in Polish).

Mazur, A. (2004). Hybrid adaptive control laws solving a path following problem for nonholonomic mobile manipulators, International Journal of Control 77(15): 1297-1306.

Nakamura, Y., Chung, W. and Sørdalen, O. J. (2001). Design and control of the nonholonomic manipulator, IEEE Transactions on Robotics and Automation 17(1): 48-59.

Samson, C. (1995). Control of chained systems-Application to path following and time-varying point-stabilization of mobile robots, IEEE Transactions on Automatic Control 40(1): 147-158.

Tan, J., Xi, N. and Wang, Y. (2003). Integrated task planning and control for mobile manipulators, International Journal of Robotics Research 22(5): 337-354.

Tchoń, K. and Jakubiak, J. (2004). Acceleration-driven kinematics of mobile manipulators: An endogenous configuration space approach, in J. Lenarčič and C. Galletti (Eds.), On Advances in Robot Kinematics, Kluwer Academic Publishers, Dordrecht, pp. 469-476.

Tchoń, K., Jakubiak, J. and Zadarnowska, K. (2004). Doubly nonholonomic mobile manipulators, Proceedings of the IEEE International Conference on Robotics and Automation, New Orleans, LA, USA, pp. 4590-4595.

Yamamoto, Y. and Yun, X. (1994). Coordinating locomotion and manipulation of a mobile manipulator, IEEE Transactions on Automatic Control 39(6): 1326-1332.

Yamamoto, Y. and Yun, X. (1996). Effect of the dynamic interaction on coordinated control of mobile manipulators, IEEE Transactions on Robotics and Automation 12(5): 816-824.

Alicja Mazur received the M.Sc. degree in automatics and the Ph.D. degree in robotics from the Wrocław University of Technology, Wrocław Poland, in 1990 and 1996, respectively. In 1997 she was a visiting professor at the Institute for Robotics and Process Control in Brunswick Germany. She currently works as an assistant professor at the Institute of Computer Engineering, Control and Robotics, Department of Electronics, Wrocław University of Technology. Her current research interests include the dynamics and control of robotic systems, nonlinear contro theory of nonholonomic systems, geometric control theory, universal and adaptive control.

Dawid Szakiel received the M.Sc. degree in robotics from the Wrocław University of Technology, Poland, in 2008. His research activity covers nonlinear control theory applied to nonholonomic mobile manipulators, path following and trajectory tracking for different robotic objects.

Received: 16 July 2009 